\title{
563.
}

\section{NOTE ON THE TRANSFORMATION OF TWO SIMULTANEOUS EQUATIONS.}

[From the Quarterly Journal of Pure and Applied IMathematics, vol. XI. (1871), pp. 266, 267.]

WRITING in Mr Walton's equations (1) and (2)

$$
\frac{a}{d}, \frac{b}{d}, \frac{c}{d}, \frac{\alpha}{\delta}, \frac{\beta}{\delta}, \frac{\gamma}{\delta}
$$

instead of $a, b, c, \alpha, \beta, \gamma$ respectively; and putting for shortness

the equations become

$$
\begin{array}{ll}
A=b \gamma-c \beta, & F=a \delta-d \alpha, \\
B=c \alpha-a \gamma, & G=b \delta-d \beta, \\
C=a \beta-b \alpha, & H=c \delta-d \gamma
\end{array}
$$

$$
\begin{aligned}
& \frac{a(b-c)}{F}+\frac{b(c-a)}{G}+\frac{c(a-b)}{H}=0, \\
& \frac{\alpha(\beta-\gamma)}{F}+\frac{\beta(\gamma-\alpha)}{G}+\frac{\gamma(\alpha-\beta)}{H}=0 .
\end{aligned}
$$

Multiplying by $F G H$ and effecting some obvious transformations, the equations become

whence also

$$
\left.\begin{array}{l}
a A F+b B G+c C H=0 \\
\alpha A F+\beta B G+\gamma C H=0
\end{array}\right\}
$$

$$
A F^{2}+B G^{2}+C H^{2}=0
$$

Now regarding $(\alpha, \beta, \gamma, \delta)$ as the coordinates of a point in space, the equations (18) and (19) represent each of them a cone having for vertex the point $\alpha: \beta: \gamma: \delta$ $=a: b: c: d$, viz. (18) is a quadric cone, (19) a cubic cone; they intersect therefore in six lines; and it may be shown that these are

$\begin{array}{lll}\text { the line } & \alpha: \beta: \gamma=a: b: c & \text { (twice) } \\ & \beta: \gamma: \delta=b: c: d & 1 \\ " & \gamma: \alpha: \delta=c: a: d & 1 \\ " & \alpha: \beta: \delta=a: b: d & 1 \\ " \beta-\gamma: \gamma-\alpha: \alpha-\beta: \delta=b-c: c-a: a-b: d & \frac{1}{6}\end{array}$

agreeing with Mr Walton's result. 